\title{
The Effect of Patho-biological Factors on the Survival of Recurrent Breast Cancer Patients
}

DOI: $10.21859 /$ mci-supp-101

\section{Keywords:}

Recurrent Breast Cancer

Survival

Prognostic Factors

\author{
Mohammad Esmaeil Akbari ${ }^{1,}$, Marzieh Rohani Rasaf ${ }^{2}$, Nahid Nafissi \\ ${ }^{3}$, Leyla Shojaee ${ }^{3}$ \\ ${ }^{1}$ Department of Surgical Oncology, Cancer Research Center, Shahid Beheshti \\ University, Tehran, Iran \\ ${ }^{2}$ School of Public Health, Shahid Beheshti University of Medical Sciences, Tehran, \\ Iran \\ ${ }^{3}$ Department of Surgery, Iran University of Medical Sciences, Tehran, Iran \\ * Corresponding author: Mohammad Esmaeil Akbari, Department of Surgical \\ Oncology, Cancer Research Center, Shahid Beheshti University, Tehran, Iran. Tel: \\ +98-9122993809, E-mail:info@crc.ir
}

